\title{
Circadian fluctuations in the efficacy of thrombolysis with streptokinase
}

\author{
E Goldhammer, L Kharash, E G Abinader
}

\begin{abstract}
Summary
This study was designed to investigate possible diurnal fluctuations in the efficacy of thrombolysis with streptokinase and whether they follow the circadian periodicity which has already been well documented for the time of onset of acute myocardial infarction, transient myocardial ischaemia, sudden cardiac death, thrombotic stroke, and for the efficacy of thrombolysis with tissue-type plasminogen and urokinase. A total of 156 consecutive patients treated with streptokinase were studied retrospectively; success or failure of thrombolysis was determined according to accepted clinical and angiographic criteria. A definite time peak for successful thrombolysis could be detected at the late afternoon and early evening hours; between 16.00 and $20.00 \mathrm{~h}, 30.2 \%$ of all successful thrombolysis cases were observed compared with $7.0 \%$ between 20.00 and $24.00(p<0.05)$ or $10.5 \%$ between 00.00 and $04.00(p<0.05)$. Between 16.00 and $20.00 \mathrm{~h}, 75.8 \%$ of treated patients had successful thrombolysis compared to $15.2 \%$ of failed treatments and $9 \%$ equivocal results $(p<0.001)$. Multiple regression analysis showed that the independent factor with the major impact on successful reperfusion was the actual time of thrombolysis $(p=0.037)$, followed by the time delay from pain onset to streptokinase administration $(p=0.020)$, while age and gender had much lesser impact $(p=0.328$ and 0.215 , respectively) and the individual risk factors even less. These findings may have several clinical implications; dose adjustment for the time of day may be required, with higher doses during morning hours, or preference for primary coronary angioplasty in order to avoid the increase in bleeding complications related to higher doses of thrombolytic agents.
\end{abstract}

Keywords: circadian periodicity; thrombolysis; streptokinase

Cardiology, Bnei-Zion

Medical Center and

School of Medicine,

Technion, 47 Golomb

Street, Haifa 31048,

Israel

E Goldhammer

L Kharash

E G Abinader

Accepted 3 June 1999 related phenomena, including sudden cardiac death, thrombotic stroke, ${ }^{5-7}$ transient myocardial ischaemia, silent ischaemia and ST-T changes in mitral valve prolapse..$^{8-10}$ Morning hypercoagulability and hypofibrinolysis with circadian variations in circulating activated factor VII, prothrombin fragment F1+2, plasminogen activator inhibitor-1 and plasmin-plasmin inhibitor complex have been described, as well as other haemostatic and physiological factors, all of which might predispose towards enhanced clotting during morning hours. ${ }^{11-13}$ This is probably the reason for the relative resistance to thrombolysis in the early morning that has been shown for both tissue plasminogen activator (t-PA) and urokinase. ${ }^{14-16}$

This study was designed to assess possible diurnal fluctuations in the efficacy of thrombolysis with streptokinase and to see whether they follow the circadian periodicity which has already been described for the abovementioned haemostatic, physiological and ischaemic phenomena. Such findings may have several clinical implications. For example, streptokinase dose adjustment for time of day may be required, thus reducing bleeding complications if the dose is reduced in certain time intervals. On the other hand, there may be a preference for primary angioplasty in time intervals in which a higher dose of streptokinase is required due to possibly decreased efficacy.

\section{Material and methods}

Results of thrombolysis were evaluated in 156 consecutive acute myocardial infarction patients admitted to the Intensive Cardiac Care Unit during the period 1 July 1995 to 1 July 1996. The study population consisted of 121 males and 35 females, age range $32-89$ years (mean $64.47 \pm 11.79$ SD). Patients were treated with streptokinase when all clinical and electrocardiographic findings corresponded to the commonly accepted criteria for acute myocardial infarction (symptoms suggestive of myocardial ischaemia, $2 \mathrm{~mm}$ or more S-T elevation in two contiguous precordial leads or $>/=1 \mathrm{~mm} \mathrm{~S}-\mathrm{T}$ elevation in two limb leads). The data which were analysed included individual coronary risk factors (hypertension, diabetes, smoking, hyperlipaemia, family history of coronary artery disease and age), first or recurrent infarction, Q and non- $Q$ infarction, time of pain onset, time of streptokinase administration, time elapsed from pain onset to streptokinase administration, peak creatine kinase and time to peak creatine kinase, fibrinogen level at the time of streptokinase administration, time to $50 \%$ recovery of S-T segment shift, type of arrhythmia (ventricular 


\begin{tabular}{|l|}
\hline Killip classes \\
\hline Class I: no signs of congestive heart failure \\
Class II: S3 gallop and bibasilar rales \\
Class III: pulmonary oedema \\
Class IV: cardiogenic shock
\end{tabular}

Box 1

premature beats, accelerated ventricular rhythm, ventricular tachycardia, supraventricular premature beats, paroxysmal supraventricular tachycardia, paroxysmal atrial fibrillation or flutter) and their time of appearance and disappearance, Killip class (box 1), bleeding complications and infarct location.

A total of 141 patients (90.4\%) underwent coronary angiography 12 hours to 21 days following infarction, the remainder did not either because of death, patient refusal or postponed angiography for other reasons.

Thrombolysis was considered successful when concordance of clinical ${ }^{17-19}$ and angiographic parameters was found, failed when clinical and angiographic data were suggestive of failure, and equivocal in those patients whose clinical indices were suggestive of success but angiographic data were unavailable or when clinical indices were equivocal and TIMI flow in the infarct-related artery was I or II. The group with equivocal results was included in order to overcome certain methodological disadvantages originating in the fact that not all coronary angiograms were performed within the first hours or days; thus thrombotic re-occlusion of an initially early reperfused artery could have occurred or, conversely, a spontaneous late thrombolysis.

Successful thrombolysis or successful reperfusion (Group 1) was determined when it was suggested by the clinical indices, early creatine kinase peak (the creatine kinase peak is normally reached between 14 and 36 hours of onset of symptoms of infarction; in patients with successful thrombolysis it is reached within the first 12 hours $^{20}$ ), early pain disap-

Table 1 Clinical data of study population according to thrombolysis result

\begin{tabular}{llll}
\hline & Success & Equivocal & Failed \\
\hline$n$ & $88(56.4 \%)$ & $20(12.8 \%)$ & $48(30.8 \%)$ \\
Males (\%) & 60.6 & 10.7 & 28.7 \\
Females (\%) & 41.2 & 20.6 & 38.2 \\
Killip Class & $1.81 \pm 0.47$ & $1.84 \pm 0.59$ & $2.31 \pm 0.7$ \\
LVEF (\%) & $41.89 \pm 6.38$ & $42.15 \pm 6.65$ & $36.93 \pm 7.29$ \\
Anterior wall MI & 43 & 7 & 28 \\
Inferior wall MI & 41 & 13 & 19 \\
Other locations & 4 & - & 1 \\
Hypertension & 36 & 9 & 8 \\
Diabetes & 16 & 8 & 11 \\
Hyperlipaemia & 26 & 3 & 18 \\
Smoking & 38 & $7.75 \pm 4.99$ & $12.15 \pm 5.41$ \\
Time to peak CK (h) & $6.32 \pm 2.54$ & $1.48 \pm 0.47$ & $5.22 \pm 1.09$ \\
Time to 50\% S-T $\downarrow$ (h) & $1.43 \pm 0.33$ & - & 2 \\
Other risk factors & 13 & 35 & 27.1 \\
Primary VT $\left.{ }^{\star} \%\right)$ & 29.5 & - & 4.16 \\
Primary VF (\%) & 2.27 & & \\
\hline
\end{tabular}

* Including multiple and multiform ventricular premature beats

LVEF: left ventricular ejection fraction; MI: myocardial infarction; CK: creatine kinase; VT: ventricular tachycardia; VF: ventricular fibrillation pearance, early reduction of $\geqslant 50 \%$ of the ST-T segment shifts, ${ }^{18}{ }^{20}$ presence of reperfusion arrhythmias ${ }^{20}$ and Killip class I or II combined with a TIMI flow grade III in the presumed infarct-related artery in coronary angiography (TIMI flow grade 0 refers to an occluded vessel with no antegrade flow while grade III refers to an open vessel with a normal antegrade flow and grades I and II indicate intermediate flow grades). Failed thrombolysis (Group 3) was determined when the same clinical criteria failed to indicate it and TIMI flow rate was $0-I$ or the patient died. An equivocal result (Group 2) was considered in those cases in which angiographic data were missing or TIMI flow was I-II and clinical indices were not definitely indicative of success.

Success and failure of thrombolysis were assessed in either four intervals of 6 hours or eight intervals of 3 hours, starting from midnight, in order to find out whether there are circadian fluctuations in the thrombolytic potency and efficacy of streptokinase.

Baseline characteristics between groups were compared with the use of the $\chi^{2}$-test, Fischer's exact test, or a two-sample $t$-test as appropriate. Correlation between variables was performed by the Spearman rank correlation test. A multivariate regression analysis that included clinical and angiographic descriptors was performed to determine which factors correlated with success or failure in the various time periods and to evaluate the impact of the time of streptokinase administration parameters on success rate.

All values are reported as mean $\pm S D$ and a probability value of $<0.05$ was considered statistically significant. StatSoft, 'Statistica for Windows', V 5.0 A software was used for the analysis.

\section{Results}

The main efficacy results are given in table 1 . Thrombolysis was determined to have been successful in 88 patients $(56.4 \%)$, failed in 48 patients $(30.8 \%)$ and equivocal in 20 patients $(12.8 \%)$. The success rate in male patients was significantly higher than in females $(60.6 \%$ vs $41.2 \%, \mathrm{p}<0.03$, 1-tailed test), while the failure rate in males was lower than in females $(28.7 \%$ vs $38.2 \%, \mathrm{p}=\mathrm{ns})$, and the percentage of equivocal results was higher in females $(20.6 \%$ vs $10.7 \%, \mathrm{p}=\mathrm{ns})$. The mean time to peak creatine kinase was 6.32 hours in Group 1, compared with 7.75 in Group 2, $(\mathrm{p}=0.0337)$ and $12.15(p<0.0001)$ in Group 3. Mean peak creatine kinase levels were significantly higher in Group 1 (1234.28 IU/1 compared to 845 IU/1 in Group 3; $\mathrm{p}=0.008$, 2-tailed). The mean time elapsed to $50 \%$ reduction of S-T segment shift was $1.43 \mathrm{~h}$ in Group 1 compared with $5.22 \mathrm{~h}$ in Group $3(\mathrm{p}<0.01)$, left ventricular ejection fraction was $41.89 \%$ in Group 1 compared with $36.93 \%$ in Group 3, $(\mathrm{p}<0.001)$ and Killip class in Group 1 was 1.81 in comparison with 2.31 in Group $3(\mathrm{p}<0.001)$.

A definite time peak for successful thrombolysis could be detected at the late afternoon and early evening hours. When the 24-h day 
Table 2 Thrombolysis success rate in various time periods

\begin{tabular}{lccl}
\hline $\begin{array}{l}\text { Time period } \\
\text { (h) }\end{array}$ & $\begin{array}{l}\text { Early } \\
\text { reperfusion }\end{array}$ & $\begin{array}{l}\text { Failed } \\
\text { thrombolysis }\end{array}$ & $\begin{array}{l}\text { Equivocal } \\
\text { result }\end{array}$ \\
\hline $00-03$ & $6(60 \%)$ & $4(40 \%)$ & 0 \\
$03-06$ & $10(52.6 \%)$ & $7(36.8 \%)$ & $2(10.6 \%)$ \\
$06-09$ & $8(57.1 \%)$ & $5(35.7 \%)$ & $1(7.2 \%)$ \\
$09-12$ & $15(62.5 \%)$ & $6(25 \%)$ & $3(12.5 \%)$ \\
$12-15$ & $12(42.8 \%)$ & $11(39.3 \%)$ & $5(17.9 \%)$ \\
$15-18$ & $11(45.8 \%)$ & $9(37.5 \%)$ & $4(16.7 \%)$ \\
$18-21$ & $19(86.4 \%)^{\star}$ & $1(4.6 \%)^{\star}$ & $2(10 \%)$ \\
$21-24$ & $7(46.7 \%)$ & $5(33.3 \%)$ & $3(20 \%)$ \\
$00-04$ & $9(52.9 \%)$ & $8(47 \%)$ & 0 \\
$04-08$ & $12(54.5 \%)$ & $7(31.7 \%)$ & $3(13.6 \%)$ \\
$08-12$ & $18(64.3 \%)$ & $7(25 \%)$ & $3(10.7 \%)$ \\
$12-16$ & $15(39.5 \%)$ & $16(42.1 \%)$ & $7(18.4 \%)$ \\
$16-20$ & $25(75.8 \%)^{\star}$ & $5(15.2 \%)^{\star}$ & $3(9 \%)$ \\
$20-24$ & $9(50 \%)$ & $5(27.8 \%)$ & $4(22.2 \%)$ \\
\hline
\end{tabular}

$\star=\mathrm{p}<0.01$

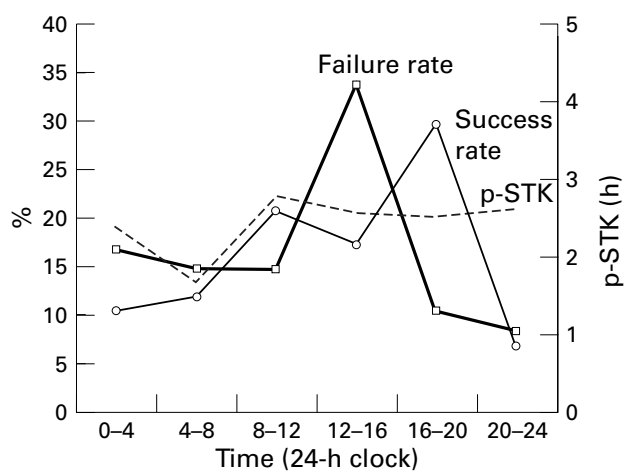

Figure 1 Thrombolysis success/failure rates and the time from pain onset to streptokinase administration (p-STK) during each 4-hour time period

was divided into eight 3-hour intervals from midnight, a peak of success was observed between $18.00-21.00 \mathrm{~h} ; 23.3 \%$ of all successful cases were observed in this time interval compared with $2.1 \%$ of all failed cases $(\mathrm{p}<0.001$, Fischer's exact test), while between 03.00$06.00 \mathrm{~h}$, the percentage of successes was $11.6 \%$ compared with $14.9 \%$ of all failures $(\mathrm{p}<0.001)$, and between $06.00-09.00 \mathrm{~h}, 9.3 \%$ successes and $10.6 \%$ failures $(\mathrm{p}=\mathrm{ns})$ (table 2$)$. When the 24-h day was divided into six 4-hour intervals (figure 1), the results were similar; in the $16.00-20.00 \mathrm{~h}$ time interval $30.2 \%$ of all

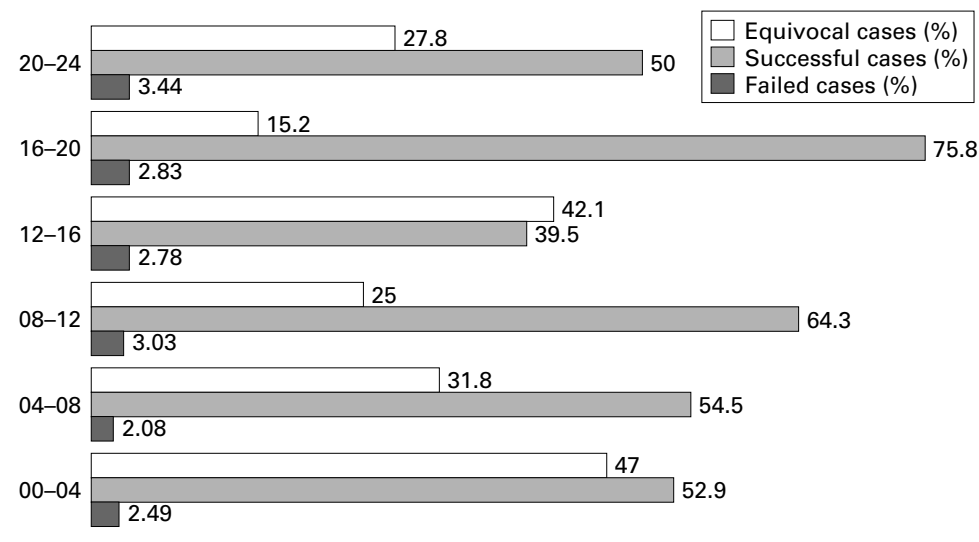

Figure 2 Thrombolysis results in each 4-hour time period successful cases of thrombolysis and only $10.6 \%$ of failures were observed $(p=0.017)$.

These findings were even more pronounced when success/failure rates were determined separately for patients treated in each time interval (figure 2); among 22 patients treated in the 18.00-21.00 $\mathrm{h}$ time interval, successful thrombolysis was determined in 19 patients $(86.4 \%)$ with one failure $(4.5 \% ; \mathrm{p}<0.001)$ and two $(9.1 \%)$ equivocal results (table 2 ). During the $16.00-20.00 \mathrm{~h}$ period the results are similar; among 33 treated patients, 25 (75.8\%) had successful thrombolysis compared with five $(15.2 \%)$ in whom thrombolysis failed and three $(9 \%)$ equivocal results.

In order to find out whether these finding could have been influenced by the most powerful determinant of thrombolysis success, the time elapsed from pain onset to streptokinase administration ( $\mathrm{p}-\mathrm{STK}$ ), this time interval was checked for each 3- and 4-hour time segment of the 24-hour period. No significant difference was found between the p-STKs in the various time intervals, which varied between $2.49 \mathrm{~h}$ \pm 1.31 and $2.83 \mathrm{~h} \pm 1.24$, except for a shorter p-STK $(2.08 \mathrm{~h} \pm 1.24)$ during early morning hours (04.00-08.00 h) and a relatively long p-STK (3.44 $\mathrm{h} \pm 1.34)$ in the late evening/early night hours $(20.00-24.00 \mathrm{~h})$. The overall success rate was influenced, as expected, by the p-STK. In the group of successful thrombolysis it was $2.47 \mathrm{~h} \pm 1.17$, compared with $3.51 \mathrm{~h}$ \pm 2.66 in the group of failed thrombolysis $(p<0.01)$. The higher success rate observed in male patients could be related to the fact that p-STK in males was shorter than in women, $2.66 \mathrm{~h} \pm 1.52$ vs $3.36 \mathrm{~h} \pm 2.59(\mathrm{p}=0.0484)$.

The percentage of male patients during the time interval of peak success rate (16.00-20.00 h) was somewhat higher than the percentage of males in the entire study group $(86.6 \%$ vs $77.56 \%, \mathrm{p}=\mathrm{ns}$ ) and it did not differ from the relative male/female percentages during other time intervals. The mean age of the group with successful thrombolysis during this period was $60.52 \pm 10.61$ years, which is quite similar to the mean age of the entire group with successful thrombolysis $(62.42 \pm 11.59$ years, $p=n s)$ but is lower than the age of patients in the group with failed thrombolysis $(66.75 \pm 13.63$ years, $p=0.0185,1$-tailed). The age difference is particularly marked when compared to the mean age of female patients in the group with failed thrombolysis $(71.62 \pm 6.58$ years, $\mathrm{p}<0.001)$.

Fibrinogen levels during the time interval 16.00-20.00 $\mathrm{h}$ were higher in patients with successful thrombolysis than in patients with failed thrombolysis $(335 \pm 82.84 \mathrm{~g} / 1$ vs $240 \pm 35$ $\mathrm{g} / \mathrm{l} ; \mathrm{p}=0.0729,1$-tailed; $\mathrm{p}=0.1457,2$-tailed). The significance of this finding remains unclear because fibrinogen levels among patients with successful thrombolysis during the the time interval $16.00-20.00 \mathrm{~h}$ is quite similar to the levels obtained in patients with failed thrombolysis during early morning hours (316.67 $\pm 69.08 \mathrm{~g} / \mathrm{l}, \mathrm{p}=\mathrm{ns})$. 


\section{Discussion}

Thrombolytic therapy with streptokinase has a definite circadian pattern of efficacy, as defined by the clinical criteria, peak creatine kinase levels, time elapsed from thrombolysis initiation to peak creatine kinase, Killip class, time to disappearance of pain, time to $50 \%$ recovery of ST segment shift, and presence of reperfusion arrhythmias, as well as TIMI flow grade III in coronary angiography. Efficacy was higher in the late afternoon and early evening hours and during these hours it was not related to the time elapsed from pain onset to thrombolysis initiation, gender, individual risk factors, or infarct location. A second peak of success, although of lesser magnitude, was noticed between 08.00 and $12.00 \mathrm{~h}$. The evidence for better efficacy in the late afternoon/early evening hours is two-fold; firstly, $30.23 \%$ of all successful thrombolytic treatments occurred in the 16.00-20.00 h period, and secondly, among patients who were treated during these hours, $86.4 \%$ had successful thrombolysis.

The reason for the relatively high percentage of failed thrombolysis (42.1\%) between 12.00 $\mathrm{h}$ and $16.00 \mathrm{~h}$, given the close proximity to the hours of peak success, remains somewhat puzzling, although it does not differ significantly from the overall failure rate $(30.8 \%)$.

These findings are concordant with the relative resistance to thrombolysis with t-PA and urokinase during morning hours and higher success rate during late afternoon/early evening hours reported by Kono ${ }^{16}$ and Kurnik ${ }^{14}$ who used the same clinical and angiographic indices for evaluation of diurnal variations in thrombolysis efficacy. Thus, the circadian variations in thrombolysis efficacy and success rate which have been shown for the most frequently used thrombolytic agents are probably independent of the type of agent used.

Chronic use of low-dose aspirin may lead to a circadian shift of acute myocardial infarction incidence, with a greater reduction during the morning waking hours. ${ }^{21}$ Acute administration just prior to streptokinase administration in our study patients could have potentiated somewhat the overall success rate of streptokinase efficacy but certainly could not have an effect on the relative success/failure rates in each time segment. Intravenous heparin was administered to all patients immediately after cessation of streptokinase and then for 72 hours, irrespective of time of admission or time of thrombolysis. Thus, the circadian effect seen with streptokinase could not be confounded by heparin.

1 Muller JE, Stone PH, Turi ZG, et al, for the MILIS group. Circadian variation in the frequency of onset of acute myocardial infarction. N Engl f Med 1985;313:1315-22.

2 WHO International Study Group. Myocardial infarction community registries: public health in Europe. Copenhagen: WHO, 1976;5:1-232.

3 Pell S, D'Alonzo CA. Acute myocardial infarction in a large industrial population: report of a 6-year study of 1356 cases. FAMA 1963;185:831-8.

4 Thompson DR, Blandford RL, Sutton TW, Merchant PR. Time of onset of chest pain in acute myocardial infarction. Int $\mathcal{F}$ Cardiol 1985;7:139-46.

5 Andreotti F, Davies GJ, Hackett DR, et al. Major circadian fluctuations in fibrinolytic factors and possible relevance to time of onset of myocardial infarction. Am $\mathcal{F}$ Cardiol time of onset
1988;62:635-7.

\section{Learning points}

- the onset of acute myocardial infarction exhibits circadian periodicity

- the early morning peak seen in transmural infarction, non-Q wave infarction and variant angina parallels the onset of other related phenomena, including sudden cardiac death, thrombotic stroke, transient myocardial ischaemia, silent ischaemia and ST-T changes in mitral valve prolapse

- circadian variations have also been described for a number of haemostatic and physiological factors, all of which might predispose towards enhanced clotting during morning hours

- there is a definite time peak for successful thrombolysis during the late afternoon/early evening period

- the clinical implications of this finding are that dose adjustment of thrombolytic agents may be required according to the time of day

Box 2

$\beta$-Blockers may influence circadian patterns of myocardial infarction pain onset, ${ }^{22}$ however only $11.54 \%$ of patients were on $\beta$-blockers at the time of streptokinase administration $(14.7 \%, 10.4 \%$ and $10 \%$ in the groups with early reperfusion, failed thrombolysis and equivocal results, respectively; $\mathrm{p}=\mathrm{ns}$ ). Therefore $\beta$-blockers did not alter the circadian pattern of streptokinase efficacy.

The a priori hypothesis for this study defined streptokinase efficacy as the variable being analysed for a circadian pattern, and thus the time of initiation of treatment is the independent variable. Multiple regression analysis showed that the independent factor with the major impact on successful reperfusion is the actual time of thrombolysis $(p=0.037)$, followed by the time delay from pain onset to thrombolysis administration $(p=0.020)$, while age and gender had a much lesser impact $(\mathrm{p}=0.328$ and 0.215 , respectively) and the individual coronary risk factors even less (smoking, $\mathrm{p}=0.213$; hypertension, $p=0.875$; diabetes, $p=0.988$; hypercholesterolaemia, $\mathrm{p}=0.622$; recurrent myocardial infarction, $\mathrm{p}=0.914)$.

These findings may have several clinical implications; dose adjustment of streptokinase according to the time of day may be required, with higher doses during morning hours, or a preference for primary angioplasty in order to avoid the increase in bleeding complications, particularly intracerebral hemorrhage, related to higher doses of thrombolytic agents.

6 Toffler GH, Brezinsky D, Schafer AI, et al. Concurrent morning increase in platelet aggregability and the risk of myocardial infarction and sudden cardiac death. $N$ Engl $\mathcal{F}$ Med 1987;316:1514-8

7 Masuda T, Ogawa H, Miyao Y, et al. Circadian variation in fibrinolytic activity in patients with variant angina. $\mathrm{Br}$ Heart f 1994;71:156-61.

8 Tzivoni $\mathrm{D}$, Circadian variations in total ischemic burden and ischemic threshold. Eur Heart f 1996;17 (suppl GP):59-63.

9 Asmar R, Benetos A, Pannier B, et al. Prevalence and circadian variations of S-T segment depression and its concomitant blood pressure changes in asymptomatic systemic hypertension. Am f Cardiol 1996;77:384-90.

10 Abinader EG, Sharif D, Goldhammer E. Attenuation of the Abinader EG, Sharif D, Goldhammer E. Attenuation of the
circadian S-T depression with propranolol in mitral valve prolapse. Am f Cardiol 1994;73:14-6. 
11 Petralito A, Mangiafico RA, Gibbino S, et al. Daily modifications of plasma fibrinogen, platelet aggregation, Howells time, PTT, TT, and antithrombin III in normal subjects and patients with vascular disease. Chronobiologia 1982;9:195-

12 Huber K, Rose D, Resch I, et al. Circadian fluctuations of plasminogen activator inhibitor and tissue plasminogen activator levels in plasma of patients with unstable coronary artery disease and acute myocardial infarction. Thromb Haemost 1988;60:372-6.

13 Kapiotis S, Jilma B, Quehenberger P, et al. Morning hypercoagulability and hypofibrinolysis. Diurnal variations in circulating activated factor VII, prothrombin fragment $\mathrm{F} 1+2$ and plasmin inhibitor complex. Circulation 1997;96:19-21.

14 Kurnik PB, Circadian variation in the efficacy of tissue type plasminogen activator. Circulation 1995;91:1341-6.

15 Becker RC, Corrao JM, Baker SP, Gore JM, Alpert JS. Circadian variation in thrombolytic response to recombinant cadian variation in thrombolytic response to recombinant
tissue-type plasminogen activator in acute myocardial tissue-type plasminogen activator in acut
infarction. 7 Appl Cardiol 1988;32:213-21.

16 Kono T, Morita H, Nishina T, et al. Circadian variations in the onset of acute myocardial infarction and efficacy of thrombolytic therapy. $\mathscr{f}$ Am Coll Cardiol 1996;27:774-8.
17 Norris RM, White HD, Cross DB, et al. Non-invasive diagnosis of arterial patency after thrombolytic treatment and its relation to prognosis. Br Heart f 1993;69:485-91.

18 Fernandez AR, Sequeira RF, Chakko S, et al. ST segment tracking for rapid determination of patency of the infarct related artery in acute myocardial infarction. $7 \mathrm{Am}$ Coll Cardiol 1995;26:675-83.

19 Langer A, Krucoff MW, Klutwijk P, et al. Non-invasive assessment of speed and stability of infarct-related artery reperfusion: results of the GUSTO S-T segment monitoring study. ₹ Am Coll Cardiol 1995;25:1552-7.

20 Hohnloser SH, Zabel M, Meinertz, Just H. Assessment of coronary artery patency after thrombolytic therapy: accurate prediction utilizing the combined analysis of three non invasive markers. F Am Coll Cardiol 1991;18:44-9.

21 Ridker PM, Manson JE, Buring JE, et al. Circadian variation of acute myocardial infarction and the effect of low dose aspirin in a randomized trial of physicians. Circulation 1990; 82:897-902.

22 Hjalmarson A, Gilpin EA, Nicod P, et al. Differing circadian patterns of symptom onset in subgroups of patients with patterns of symptom onset in subgroups of patients with
acute myocardial infarction. Circulation 1989;80:267-75.

\section{Images in medicine}

\section{Actinomycetoma pedis}

A 48-year-old woman presented with an 8-year history of recurrent, painless swellings with chronic pus discharge from her right foot after a minor injury while she was working in a

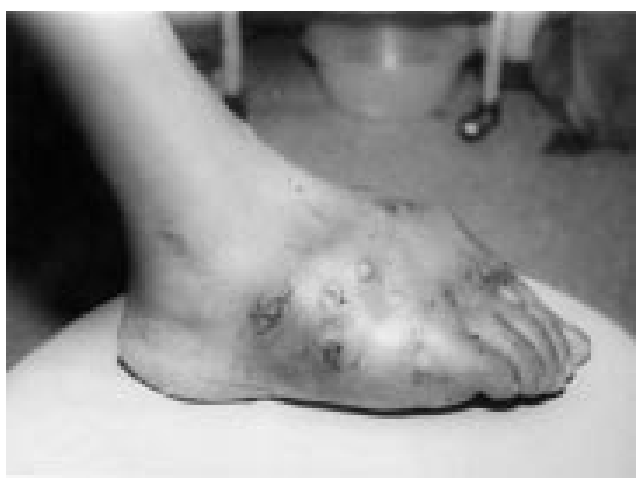

Figure 1

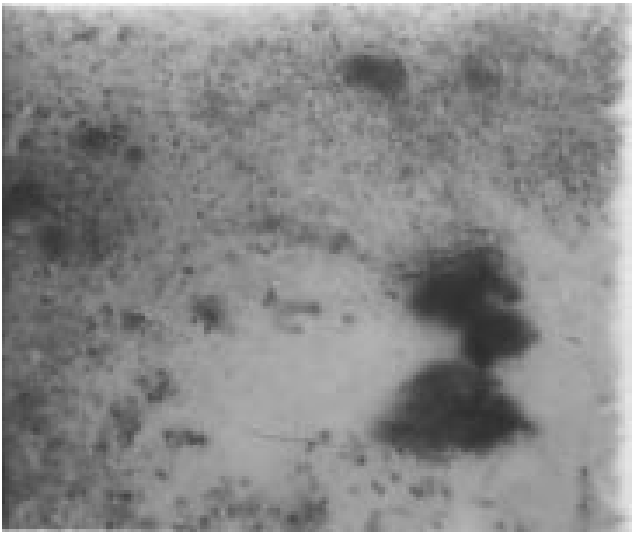

Figure 2 paddy field. There was no visceral involvement in the form of fever, organomegaly or lymphadenopathy. She had received multiple treatment modalities including 9 months of antituberculous therapy without any relief. Examination of her right foot showed multiple painless swellings and woody induration with sinus tracts (figure 1). Pus discharge from sinus tracts revealed typical grains of $2-3 \mathrm{~mm}$ diameter. Tissue Gram staining showed fine branching hyphae of Actinomadura within actinomycetoma grain (figure 2). X-Ray of the foot showed destruction of metatarsal bones with multiple cavities (figure 3). The lesions have shown definite regression after 6 months of therapy with dapsone and streptomycin.

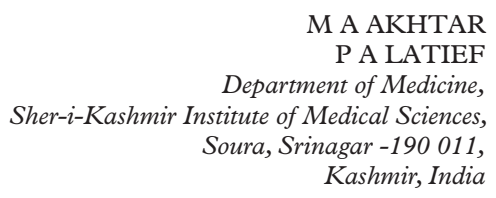

Accepted 3 June 1999

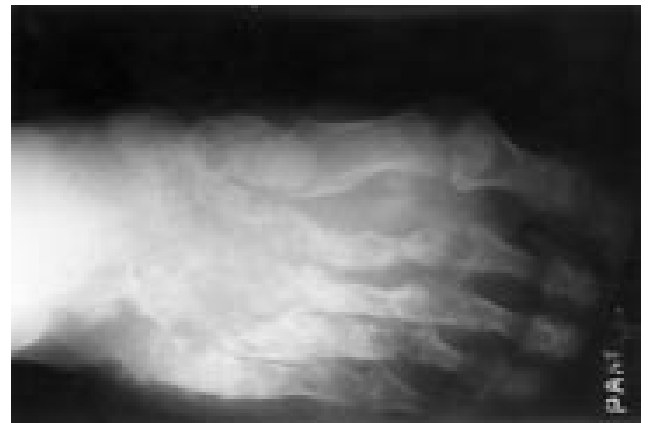

Figure 3 